\title{
Brown-Vialetto-Van Laere and Fazio Londe syndromes: defects of riboflavin transport with biochemical similarities to multiple acyl-CoA dehydrogenation defects (MADD)
}

\author{
See original article DOI 10.1007/s10545-012-9513-y
}

\author{
Michael J. Bennett
}

Received: 31 May 2012 /Revised: 31 May 2012 / Accepted: 6 July 2012 /Published online: 14 September 2012

(C) SSIEM and Springer 2012

Riboflavin-responsive defects of electron transfer were first described in the 1980s as mild variant forms of MADD or glutaric acidemia type II (Gregersen et al 1982; Amendt and Rhead 1986). Some of these patients were shown to have defects in ETF and ETF dehydrogenase but a number of patients remained in whom the biochemical and molecular defects remained elusive (Roettger et al 1992). It was postulated that these patients may have $\operatorname{defect}(\mathrm{s})$ of riboflavin transport or abnormalities in downstream flavin metabolism to form the active FAD/FMN cofactors required for facilitating electron transfer. Treatment of some of these patients with high dose riboflavin tended to normalize the abnormal metabolite profiles. Treatment with high dose riboflavin was also subjectively claimed to improve the clinical phenotype, a difficult judgment given the mild and intermittent nature of the phenotype. The phenotype of most of the cases of riboflavin-responsive MADD was that of stress-induced myopathy (de Visser et al 1986; DiDonato et al 1989).

Recently, the genetic basis for Brown-Vialetto-Van Laere syndrome (BVVLS) was identified as a previously unknown open reading frame C20orf54 (Green et al 2010). This gene was subsequently shown to encode a riboflavin transporter and the gene is now renamed SLC52A3 encoding human riboflavin transporter 2 (hRFT2). At around the same time, a dominantly inherited mutation in SLC52A1 encoding human riboflavin transporter 1 (hRFT1) was identified in the

Communicated by: K. Michael Gibson

M. J. Bennett $(\bowtie)$

Department of Pathology \& Laboratory Medicine,

Children's Hospital of Philadelphia,

34th Street \& Civic Center Blvd,

Philadelphia, PA 19104, USA

e-mail: bennettmi@email.chop.edu clinically normal mother of a neonate with complex neurological disease and a MADD metabolite profile which responded dramatically to riboflavin therapy. It is presumed that the neonate was riboflavin deficient secondarily to the maternal haploinsufficiency and from increased riboflavin demand during pregnancy (Ho et al 2011). Bosch and coworkers and others extended the phenotype of SLC52A3 defects to include patients with Fazio Londe syndrome, which was previously considered to be an overlapping clinical phenotype but without a molecular basis (Bosch et al 2011). Anand and coworkers added an additional riboflavin-responsive BVVLS case to the data base (Anand et al 2012). In this volume of $J I M D$, Haack et al 2012, demonstrating the power of whole exome sequencing identified disease-causing mutations in a third riboflavin transporter in an individual with BVVLS. They identified mutations in SLC52A2 and exquisitely demonstrate a functional defect of hRFT3, the gene product. This present case also highlights the likely mechanisms of disease phenotype in BVVLS.

The clinical phenotype of BVVLS is not what we would immediately associate with defects of electron transfer such as MADD. For this reason, it is important for investigators to recognize this phenotype as it would appear that these defects of riboflavin transport are eminently treatable with high dose riboflavin therapy. BVVLS is rare and has been reported in about 60 patients in total but may be under diagnosed. It presents primarily with a severe sensorineural deafness. The age of onset is from infancy to the third decade. During the relentless progress, lower motor neuron limb signs lead to neuropathy and are accompanied by cerebellar ataxia, optic atrophy, retinal pigmentary changes, mental retardation and psychiatric abnormalities. Death is usually a result of diaphragmatic paralysis (Dipti et al 2005). 
Many of these features are also seen in amyotrophic lateral sclerosis. Fazio-Londe syndrome has many of the same features but does not have the associated deafness. Other overlapping rare syndromes include Madras motor neuron disease, Boltshauser syndrome and Nathalie syndrome.

Despite, not having an obvious clinical correlate with MADD, defects of both SLC52A3 and now SLC52A2 both present with biomarkers that suggest a defect of electron transfer from multiple acyl-CoA species. Patients with defects of SLC52A3 presented with abnormal plasma acylcarnitines of chain lengths $\mathrm{C} 4-\mathrm{C} 14$ prior to riboflavin therapy $(10 \mathrm{mg} / \mathrm{kg}$ body weight/day), all of which normalized on therapy. These patients had modestly abnormal urine organic acids, primarily with ethylmalonate, adipate and 2hydroxyglutarate consistent with the reported profiles for ethylmalonic-adipic aciduria rather than glutaric aciduria type II. Plasma levels of all flavins were very low prior to treatment and normalized on treatment. All three of the patients reported by Bosch et al had remarkable responses to the riboflavin, two of whom are now completely normal. The single case report presented with a SCL52A2 defect by Haack and coworkers in this issue of the journal demonstrated normal urine organic acids and plasma flavin levels unlike the SLC52A3 mutants but there were similarly abnormal plasma acylcarnitines in this patient which suggested the possibility of impaired electron transfer. The patient was started on high dose riboflavin therapy and over a very short period of monitoring did have marked improvement in gait and motor skills and correction of the acylcarnitine abnormalities.

Tissue expression of hRFT 1 and hRFT2 is predominantly in the small intestinal epithelial cells, indicating that the low levels of circulating flavins in patients with SCL52A1 and $S C L 52 A 3$ are a result of failure to absorb riboflavin from the diet, explaining the very low circulating levels. These two transporter proteins may be complimentary; in particular hRFT2 may transport sufficient riboflavin to prevent profound riboflavin deficiency when there is haploinsufficiency of hRFT1. hRFT3 is primarily expressed in the brain explaining the normal circulating levels of flavins but there is impaired transport of riboflavin into the brain in hRFT3 deficiency resulting in lack of flavins for normal brain metabolism. This may account for the significant neurological signs and symptoms that are seen in BVVLS patients.

BVLLS should theoretically be a diagnosable condition via acylcarnitine measurement in newborn screening programs as there is a characteristic profile. However, at this time it is not known if newborns with this condition are initially protected by transfer of maternally-derived riboflavin. The clear benefit of high dose riboflavin therapy in an otherwise relentless neurological decline makes this condition an important one for all of us to be aware of and to hopefully recognize and treat accordingly.

Conflict of Interest None.

\section{References}

Amendt BA, Rhead WJ (1986) The multiple acyl-coenzyme A dehydrogenation disorders, glutaric aciduria type II and ethylmalonicadipic aciduria. J Clin Investig 72:208-213

Anand G, Hasan N, Jayapal S et al (2012) Early use of high-dose riboflavin in a case of Brown-Vialetto-Van Laere syndrome. Dev Med Child Neurol 54:187-189

Bosch AM, Abeling NG, Ijlst L et al (2011) Brown-Vialetto-Van Laere and Fazio Londe syndrome is associated with a riboflavin transporter defect mimicking mild MADD: a new inborn error of metabolism with potential treatment. J Inherit Metab Dis 34:159-164

De Visser M, Scholte HR, Schutgens RBH et al (1986) Riboflavinresponsive lipid storage myopathy and glutaric aciduria type II of early adult onset. Neurology 36:367-372

DiDonato S, Gellera C, Peluchetti D et al (1989) Normalization of short-chain acylcoenzyme A dehydrogenase after riboflavin treatment in a girl with multiple acylcoenzyme A dehydrogenasedeficient myopathy. Ann Neurol 25:479-484

Dipti S, Childs A, Livingstone JH et al (2005) Brown-Vialetto-Van Laere syndrome; variability in age of onset and disease progression highlighting the phenotypic overlap with Fazio-Londe disease. Brain Dev 27:443-446

Green P, Wiseman M, Crow YJ et al (2010) Brown-Vialetto-Van Laere syndrome, a pontobulbar palsy with deafness is caused by mutations in c20orf 54. Am J Hum Genet 86:485-489

Gregersen N, Wintzensen H, Kolvraa S et al (1982) C6-C10 dicarboxylic aciduria: investigations of a patient with riboflavin responsive multiple acyl-CoA dehydrogenation defects. Pediatr Res 16:861868

Haack TB, Makowski C, Yao Y, et al (2012) Impaired riboflavin transport due to missense mutations in SLC52A2 cause BrownVialetto-Van Laere syndrome. J Inherit Metab Dis. doi:10.1007/ s10545-012-9513-y

Ho G, Yonezawa A, Masuda S et al (2011) Maternal riboflavin deficiency resulting in transient neonatal-onset glutaric aciduria type 2 is caused by a microdeletion in the riboflavin transporter gene GPR172B. Hum Mutat 32:E1976-E1984

Roettger V, Marshall T, Amendt B, Rhead WJ (1992) Multiple acyl coenzyme A dehydrogenation disorders (MAD) responsive to riboflavin: biochemical studies in fibroblasts. Prog Clin Biol Res 375:317-326 